\title{
Vorkommen von Vanadium im Grundwasser der Vulkaneifel
}

\author{
Linda M. Härter ${ }^{1} \cdot$ Michael Kersten ${ }^{1} \cdot$ Andreas Riße $^{2} \cdot$ Rudolf Poppe $^{2} \cdot$ Georg Wieber $^{2}$
}

Eingegangen: 4. Juni 2019 / Überarbeitet: 10. Dezember 2019 / Online publiziert: 18. März 2020

(c) Der/die Autor(en) 2020

\section{Zusammenfassung}

In Quellwasserproben rund um den Ringseitert-Vulkankomplex südöstlich der Gemeinde Kirchweiler (Westeifel) wurden weit über dem Geringfügigkeitsschwellenwert erhöhte Vanadiumkonzentrationen mit einem Maximalwert von $28 \mu \mathrm{g} / 1$ nachgewiesen. Generell zeigten die im Bereich der Känozoischen Vulkanite entnommenen Wasserproben erheblich höhere Konzentrationen als die im umliegenden Paläozoikum. Lokale Vulkanitproben weisen bis zu vierfach über dem Krustenmittel erhöhte Gesamtgehalte an Vanadium auf. Elutionsuntersuchungen ergaben eine erhöhte Vanadiumfreisetzung. Zur weiteren mikroanalytischen Suche nach der geogenen Quelle für diese Anomalie wurden Dünnschliffe der Gesteinsproben hergestellt und mittels Elektronenmikrosonde analysiert. Die Elementverteilungsbilder zeigten nicht die erwartete Korrelation zwischen den Elementen Fe und V, wohl jedoch eine Korrelation zwischen P und V. Hotspot-Analysen von V-reichen Mineralkörnern weisen auf das Mineral Fluorapatit mit bis zu 5 Gew.-\% Vanadat als Substitution für das Phosphat als geogene Vanadiumquelle in den Vulkaniten hin. Eine hydrogeochemische Modellierung mit PhreePlot zeigt übereinstimmend, dass die Wasserproben mit erhöhten Vanadiumkonzentrationen alle im pe/pH-Prädominanzfeld der Vanadat(V)-Komplexe liegen.

\section{Vanadium in groundwater of the Eifel volcanic area, Germany}

\section{Abstract}

Elevated vanadium concentrations of up to $28 \mu \mathrm{g} / \mathrm{l}$ were found in spring waters of the western Eifel volcanic area, Germany. The spatial distribution of the water samples indicates higher $\mathrm{V}$ concentrations in Cenozoic vulcanite than in neighboring Paleozoic areas. X-ray fluorescence analyses of the volcanic rock samples revealed a $\mathrm{V}$ enrichment up to four-fold above average earth crust values. Water elution tests showed enhanced mobility of the $\mathrm{V}$ inventory. Microprobe element mapping with thin slices of the volcanic rocks showed no correlation between Fe and V but revealed a spatial correlation between $\mathrm{P}$ and V. Microprobe chemical analysis of V-rich hot spots revealed V-bearing fluorapatite with up to $5 \%$ of the phosphate substituted by vanadate. Fluorapatite micro-grains are abundant in the rocks and may well represent a geogenic source for the $\mathrm{V}$ contamination of the groundwater. Hydrogeochemical modeling using PhreePlot shows the vanadate (V) species which dominate the V-enriched water samples.

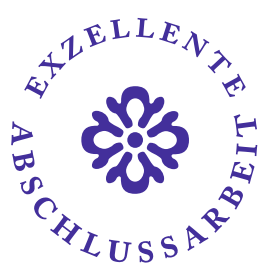

Keywords Volcanic aquifer $\cdot$ Vanadium mobility $\cdot$ Vanadate $\cdot$ V-bearing Fluorapatite

Linda M. Härter

linda.haerter@gmx.de

Michael Kersten

kersten@uni-mainz.de
1 Institut für Geowissenschaften, Johannes Gutenberg-Universität Mainz, J.-J. Becherweg 21, 55128 Mainz, Deutschland

2 Landesamt für Geologie und Bergbau Rheinland-Pfalz, Emy-Roeder-Straße 5, 55129 Mainz, Deutschland 


\section{Einleitung}

Gemäß EG-Grundwasserrichtlinie vom 20. Juni 2014 sind hydrogeochemische Hintergrundwerte in Abhängigkeit vom Gesteinsinventar der jeweiligen Grundwasserleiter zu ermitteln. Für eine Zustandsbewertung der Grundwasserkörper müssen zudem sowohl anthropogene wie auch geogene Prozesse zur Beeinflussung der Grundwasserqualität besser verstanden werden (Burri et al. 2019). Als toxische Spurenelemente natürlichen (geogenen) Ursprungs in Grundwässern sind unter anderem Oxoanionen der Elemente Arsen und Antimon allgemein bekannt. Vanadium liegt im Grundwasser üblicherweise ebenfalls in Form des Oxoanions Vanadat vor, allerdings in meist sehr geringen Konzentrationen (Huang et al. 2015). Im Jahr 2014 wurde von der LAWA eine Neuauswertung aller bisher gewonnenen Hintergrundwerte vorgenommen (Wagner et al. 2011). Der nationale Basiswert als flächengewichtetes Mittel der 90. Perzentilwerte der Hintergrundwerte aller Bundesländer wurde in dieser Studie mit 1,7 $\mu \mathrm{g} / \mathrm{l}$ bestimmt (Bergmann et al. 2015). Der Geringfügigkeitsschwellenwert (GFSWert) für Vanadium wurde mit $4 \mu \mathrm{g} / \mathrm{l}$ etwa doppelt so hoch angesetzt (LAWA 2004, 2017). Dieser GFS-Wert impliziert jedoch keinen toxikologischen Schwellenwert zur Risikobewertung, sondern lediglich eine Grundlage zur Erkennung von Konzentrationsanomalien. In der deutschen Trinkwasserverordnung ist Vanadium nicht mit einem Grenzwert belegt und unterliegt somit keiner Beschränkung.

Für das Verständnis der Mobilität von Vanadium im Grundwasser sind dennoch in letzter Zeit erhebliche Forschungsanstrengungen unternommen geworden, die sich insbesondere mit der Interaktion zwischen den verschiedenen gelösten V-Spezies und potenziellen Wirtsphasen in den Aquifergesteinen befassen. Im Gegensatz zu Arsen treten hohe Vanadiumkonzentrationen vornehmlich in sauerstoffreichen und alkalischen Wässern auf (Gustafsson 2019). Erhöhte Vanadiumkonzentrationen von bis zu $180 \mu \mathrm{g} / \mathrm{l}$ wurden weltweit vornehmlich aus Regionen mit Vulkaniten wie z.B. vom Ätna und ebenso dem Mt. Fuji berichtet (Hamada 1998; Minelli et al. 2000; Arena et al. 2015; Koh et al. 2016). Die fortschreitende Verwitterung und Auflösung von V-haltigen mafischen Mineralen der vulkanischen Gesteine wird als Hauptquelle erhöhter Vanadiumkonzentrationen in den Wässern angesehen (Huang et al. 2015; Hyon et al. 2016). In der HÜK200 der BGR (http://www.bgr.de/Service/grundwasser/huek200/ hgw/) fallen bei Darstellung des 90. Perzentils erhöhte Werte im Bereich der tertiären Vulkanite des Vogelsbergs mit 8,4 $\mu \mathrm{g} / \mathrm{l}$ sowie in den känozoischen Basalten und Tuffen des Westerwaldes mit sogar $11,9 \mu \mathrm{g} / \mathrm{l}$ auf, also dreimal so hoch wie der GFS-Wert (Bergmann et al. 2015). In Wasserproben eines Trockenmaares der Westeifel wurden im Rahmen dieser Studie ebenfalls erhöhte Vanadiumkonzen- trationen festgestellt. Es scheint also ein Charakteristikum von Grundwasserleitern in Vulkangebieten zu sein, dass die Vanadiumkonzentrationen geogen erhöht sind. Ziel dieser Studie war es, die geogene Quelle für diese erhöhten Vanadiumkonzentrationen $\mathrm{zu}$ identifizieren. Verschiedene Hypothesen wurden unter Bezugnahme auf die anstehenden Gesteine und ihrer Mineralogie betrachtet. Für eine Quantifizierung der Wechselwirkung zwischen Gesteinsmatrix und Grundwasser wurden Elutionsuntersuchungen, Mikrosondenanalysen sowie thermodynamische Gleichgewichtsrechnungen durchgeführt. Mithilfe dieser hydrogeochemischen Untersuchungen wurden eine potenzielle Wirtsphase erkannt sowie die Speziation und Mobilität des Vanadiums quantifiziert.

\section{Hydrogeologie der Trockenmaare in der Westeifel}

Im quartären Vulkanismus der Westeifel wurden Lavaströme, Schlackenkegel, Tuffwälle und zahlreiche Maare gebildet. Allein in der Westeifel sind über 256 Eruptionszentren und rund 98 Maare bekannt (Seib et al. 2013). Der weitaus größte Teil befindet sich entlang eines $50 \mathrm{~km}$ langen, von Ormont bis Bad Bertrich erstreckenden, NW-SEstreichenden Feldes (Lange et al. 2019). Das posteruptive Entwicklungsstadium eines Maars zeichnet sich durch die starke morphologische Veränderung des Kraters aus. Die meisten Maare wurden konzentrisch mit Auswurfsmaterial und Sediment verfüllt, verlandeten und wurden schließlich zu Trockenmaaren (Weiler 2002). Trockenmaare unterscheiden sich aufgrund ihrer Verfüllung gravitativ von ihrer Umgebung und konnten mithilfe geophysikalischer Messungen erkannt und vermessen werden (Weiler 2002; Donndorf 2009; Hesse 2002). In den gering wasserwegsamen devonischen Umgebungsgesteinen der Klerf-Schichten (Wechsellagerung von Ton-, Sand- und Siltsteinen) stellen die Maare kleinräumige, aber ergiebige Grundwasserreservoire von großer wasserwirtschaftlicher Bedeutung für die überwiegend dezentral organisierte Wasserversorgung der Eifelregion dar. Häufig ergeben sich daraus Nutzungskonflikte zwischen Grundwassererschließung, intensiver Landwirtschaft sowie Baustoffgewinnung. Quellaustritte in den Randbereichen eines der Trockenmaare wurden im Rahmen dieser Studie für eine Beprobung ausgewählt.

Der nordwestlich von Daun und südöstlich der Gemeinde Kirchweiler gelegene mafische Ringseitert-Vulkanitkomplex wird durch ein zentrales, von Schlackenkegeln umsäumtes Trockenmaar dominiert. Mit einer Fläche von nur $2,5 \mathrm{~km}^{2}$ ist der Ringseitert eines der größeren bekannten Trockenmaare der Westeifel (Weiler 2002). Aufgrund seiner Größe und internen Struktur weist es hervorragende Eigenschaften zur Grundwasserneubildung auf. Die 
durchschnittlichen Grundwasserneubildungsraten im untersuchten Gebiet belaufen sich nach dem Hydrogeologischen Atlas Rheinland-Pfalz auf $<50 \mathrm{~mm} / \mathrm{a}$ bis über $125 \mathrm{~mm} / \mathrm{a}$ (LUWG 2005). Die Grundwasserneubildung in Maaren unterscheidet sich jedoch deutlich gegenüber ihrer Umgebung. Trockenmaare sind in der Regel mit Pyroklastika und Sedimentmaterial von sehr guten Wasserspeichereigenschaften verfüllt. Der im Schlot befindliche periglaziale Solifluktionsschutt, Tuffaschen sowie phreatomagmatische Tephra zeichnen sich durch eine hohe Porosität gegenüber dem devonischen Grundgebirge aus. Aufgrund des größtenteils fehlenden Feinkornanteils weisen diese Porengrundwasserleiter allerdings ein nur geringes Filtervermögen auf. Zumindest aber sorgt eine bis mehrere Meter mächtige Deckschicht von bimsaschereichen Solifluktionsböden (Andosol gemäß WRB-Klassifikation) für einen gewissen Grundwasserschutz gegenüber landwirtschaftlicher Beeinträchtigung. Aufgrund der relativ guten Durchlässigkeit der pyroklastischen Schlotfüllung und mithin schneller Versickerung kann die reale Verdunstung geringer angenommen werden als im Hydrologischen Atlas von RheinlandPfalz für das Gebiet angegeben wird. Auch stellt die naturräumliche Struktur eines Maares einen hydraulischen Entlastungspunkt dar und fördert das Anströmen von Oberflächenwässern aus der Umgebung (Hesse 2002; Weiler 2002). Köppen (1991) beschreibt dementsprechend eine Grundwasserneubildung für die Aquifere der benachbarten Trockenmaare Booser Maar, Drees-Dottingen und Hausten-Morswiesen mit überdurchschnittlichen Werten von 3,5-6,0 1/s $\cdot \mathrm{km}^{2}(\sim 110-180 \mathrm{~mm} / \mathrm{a})$.

\section{Methodik}

Für die vorliegende Arbeit wurden öffentlich zugängliche Quellaustritte beprobt (Abb. 1, Tab. 1). Die Entnahmestandorte befinden sich innerhalb des Trockenmaares und der benachbarten Vulkanstruktur. Eine detaillierte Beschreibung der Probennahmestandorte mit den aufgenommenen Feldparametern und Quellenmerkmalen sind bei berechtigtem Interesse der unveröffentlichten Masterarbeit zu entnehmen (Härter 2018). Die Wasserproben wurden mithilfe von PESpritzen und Spritzenvorsatzfilter in die Probenflaschen überführt. Die PE-Spritzen wurden dazu mehrfach mit dem zu beprobenden Wasser vorkonditioniert und anschließend durch den ebenfalls vorkonditionierten Membranfilter (Typ Minisart CA 0,2 $\mu \mathrm{m}$, Sartorius) in die jeweiligen Probenflaschen mit darin enthaltenen $200 \mu 1 \mathrm{HNO}_{3}$ (MERCK suprapur) filtriert. Zusätzlich wurde darauf geachtet, dass die Proben bis zur Analyse kühl, dunkel und geschützt vor äußeren Einflüssen gelagert wurden. Für die Analyse der Kationen- sowie Spurenelementgehalte wurden im Labor des Landesamtes für Geologie und Bergbau (LGB) Mainz ein ICP-MS-Gerät des Typs ELAN-DRC-e (Axial Field Technology) der Firma Perkin Elmer SCIEX gemäß DIN EN ISO 17294-2:2005-02 verwendet. Zur Analysenqualitätskontrolle wurden hausinterne Standards in verdünnter $\mathrm{HNO}_{3}$ (MERCK suprapur) angefertigt. Die Messgenauigkeit für Vanadium wurde mit einem Merck-Standard überprüft. Der prozentuale Fehler der Standardmessung lag bei $0,2 \%$ und die Bestimmungsgrenze bei $0,03 \mu \mathrm{g} / \mathrm{l}$. Die Standardabweichung wurde mittels 4-fach Messung jeder Probe errechnet. Die Chlorid-, Nitrat- und Sulfatkonzentrationen wurden gemäß DIN EN ISO 10304-1 mithilfe der Flüssigkeits-Ionenchromatographie (Metrohm 930) bestimmt. Die Phosphatkonzentration wurde gemäß DIN EN ISO 6878 mit der Ammoniummolybdat-Methode untersucht. Die Nachweisgrenze für das Verfahren wurde gemäß DIN 32645 mit $10 \mu \mathrm{g} / 1$ bestimmt.

Gesteinsdünnschliffe wurden von Gesteinshandstücken angefertigt, die in Aufschlüssen der Region (Probennahmelokationen siehe Abb. 2) herausgebrochen wurden, und mittels klassisch petrographischer sowie mikroanalytischer Methoden untersucht. Für die petrographischen Analysen wurden ein Polarisationsmikroskop Orthoplan der Fa. Leica mit DFC 450 Digitalkamera verwendet und in Verbindung mit der mitgelieferten Software LAS (Leica Application Sweep V. 3.8) die Mineralparagenese quantifiziert (Härter 2018). Für die mikroanalytisch-chemischen Untersuchungen (,element mapping') der Dünnschliffe wurde eine Elektronenmikrosonde (Jeol JXA 8200) eingesetzt.

Die beprobten Gesteinshandstücke entsprachen jedoch nicht den nach DIN 19529 geforderten Korngrößen bei der Elutionsuntersuchung zur Ermittlung des mobilisierbaren Schwermetallanteils und mussten dafür zunächst zerkleinert werden. Bedingt durch die angestrebte Analyse auf Vanadium wurden ausschließlich Mahlwerkzeuge aus Achat und Wolframkarbid gewählt, um eine Kontamination durch Geräteabnutzung zu minimieren. Die Vorzerkleinerung wurde im Achatmörser vorgenommen. Im Anschluss wurde das zerkleinerte Material händisch ausgelesen und stark angewitterte Teile aussortiert. Das übrige Material wurde in eine Scheibenschwingmühle aus Wolframkarbid gegeben und das so erhaltene Mahlgut trotz der dann deutlich kleineren Partikelgröße als in DIN 19529 empfohlen für die Elutionsuntersuchung verwendet. Die angesetzte Gesteinspulvermenge belief sich auf $100 \mathrm{~g}$ Trockenmasse. Die mittels BET-Gerät gemessenen spezifischen Oberflächen lagen zwischen 2 und $20 \mathrm{~m}^{2} / \mathrm{g}$ (Härter 2018). Die Proben wurden mit $200 \mathrm{ml}$ Milli-Q Reinstwasser in $500 \mathrm{ml}$ PET-Flaschen gegeben und $24 \mathrm{~h}$ im Überkopfschüttler bei 20 Umdrehungen pro Minute behandelt. Im Anschluss wurde die Probe 15 min ruhen gelassen, damit sich Schwebstoffe absetzen konnten. Der klare Überstand wurde abdekantiert und $30 \mathrm{~min}$ bei $2000 \mathrm{~g}$ zentrifugiert. Die zentrifugierte Probe wurde abschließend noch membranfiltriert (Cellulosefilter 


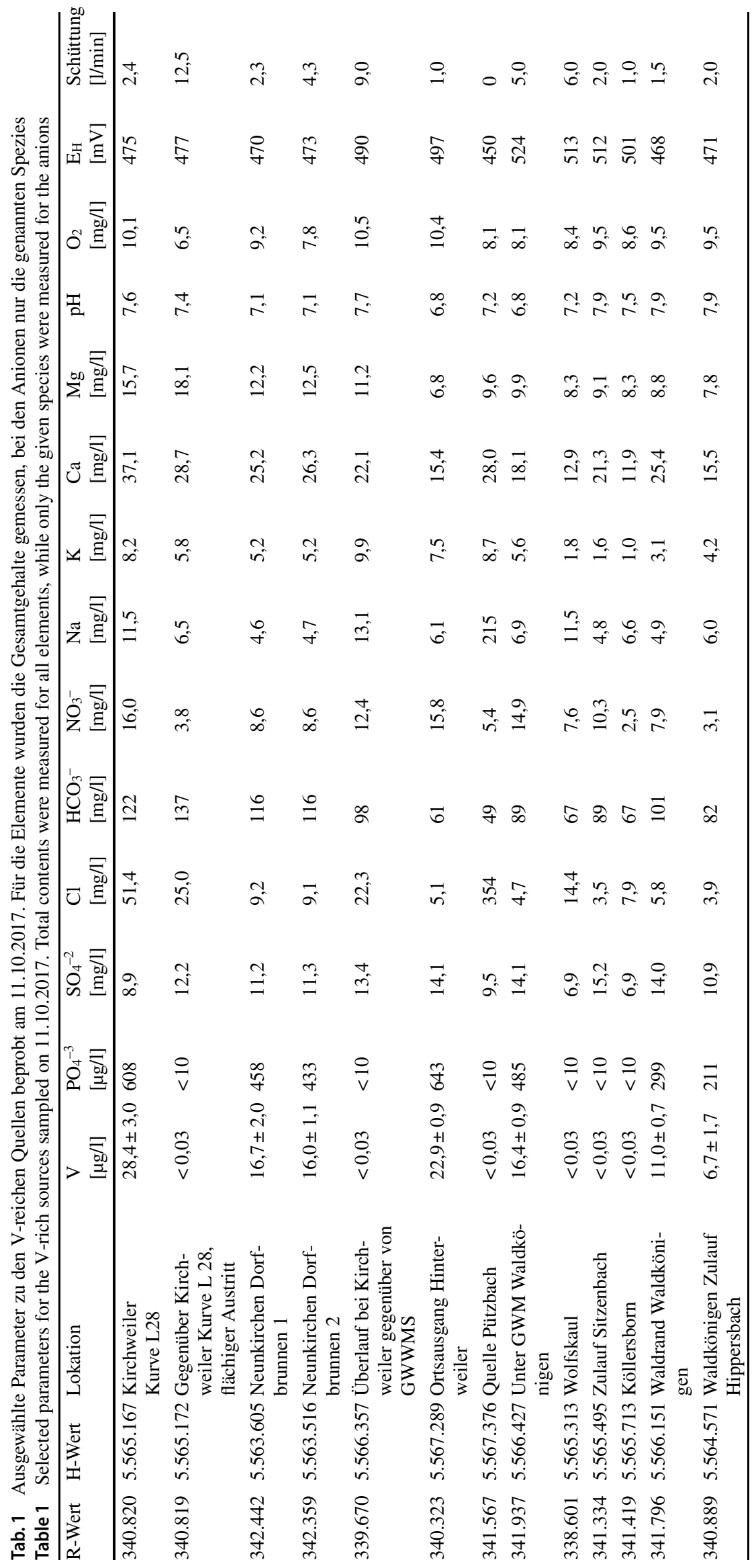




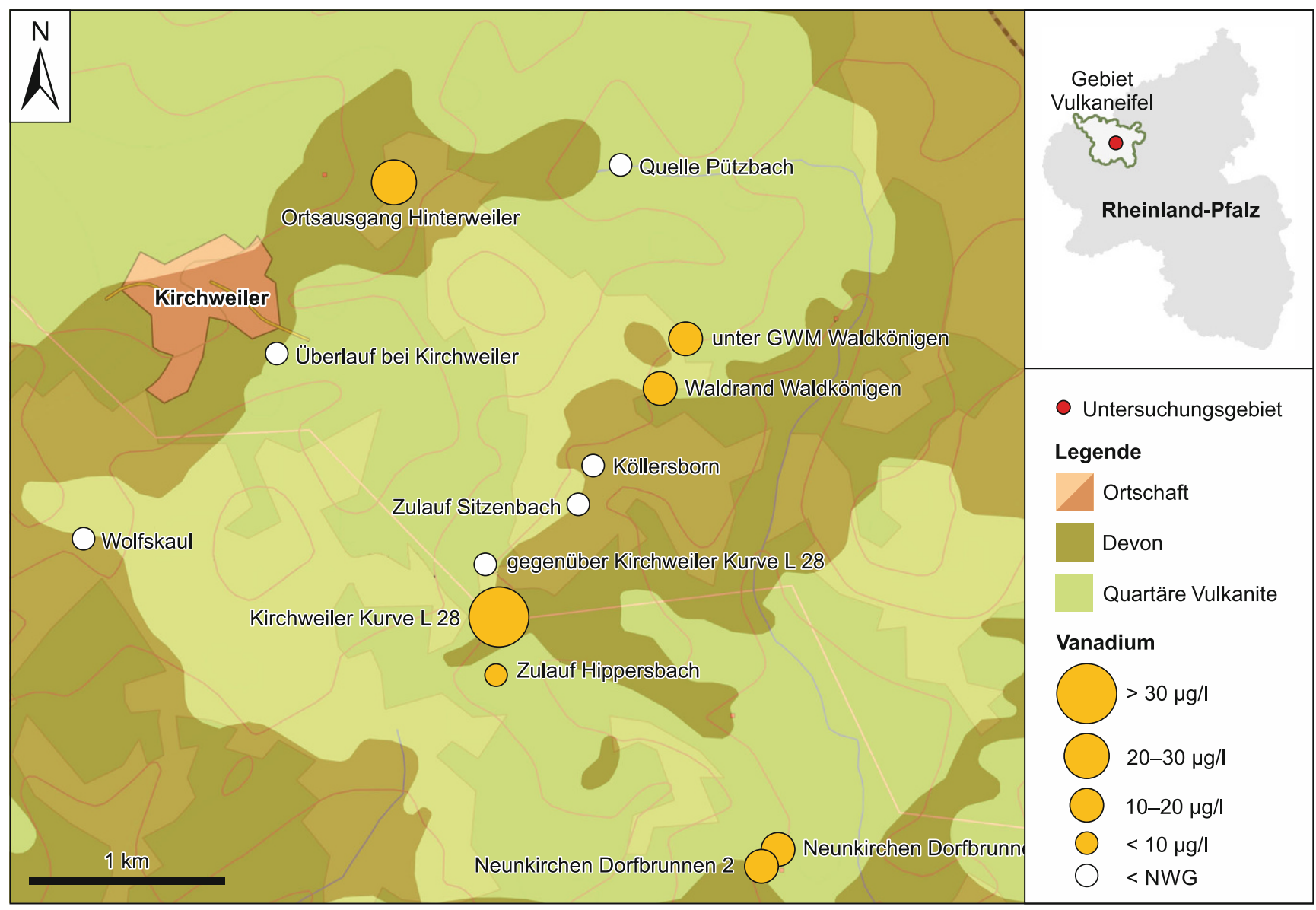

Abb. 1 Konzentrationsverteilung des gelösten Vanadiums in den beprobten oberflächlichen Quellaustritten der Ringseitert-Vulkanstruktur bei Kirchweiler. Die kleine Karte zeigt Rheinland-Pfalz mit der Markierung zur Lage der Vulkanstruktur in der Westeifel

Fig. 1 Concentration distribution of dissolved vanadium in the sampled spring outlets of the Ringseitert volcano structure near Kirchweiler. The map shows Rhineland Palatinate with a mark for the location of the volcano structure in the W-Eifel region

Minisart CA 0,2 $\mu \mathrm{m}$, Sartorius). Nachdem der erste Probensatz entnommen wurde, wurden die residualen Feststoffproben mit $200 \mathrm{ml}$ Reinstwasser aufgefüllt und erneut für insgesamt $94 \mathrm{~h}$ in den Überkopfschüttler gegeben. Nach Ablauf der 94h wurden nach dem gleichen Verfahren Elutionsproben hergestellt. Als Blindprobe diente laborreiner Quarzsand, der in allen Versuchsschritten identisch zu den Gesteinsproben behandelt wurde. Die Eluate wurden schließlich angesäuert und mithilfe der ICP-MS (Agilent 7700) auf die Vanadiumkonzentrationen analysiert. Die V-Gehalte der Gesteinspulver wurden mittels Röntgenfluoreszenzanalytik (RFA ARL PERFORM'X, Thermo Fisher) bestimmt.

\section{Ergebnisse und Diskussion}

\section{Wasserproben}

Die physikochemischen Parameter sowie der Chemismus aller Wasserproben sind der Tab. 1, deren Typisierung ge- mäß Piper- und Schöller-Diagrammen sowie weitere Spurenelementkonzentrationen der Masterarbeit zu entnehmen (Härter 2018). Die Plausibilität der Analysenergebnisse zum Chemismus aller Wasserproben wurde anhand der Ionenbilanz überprüft, die im Toleranzbereich (max. 5,7\% Differenz) nach Langguth und Voigt (2004) lag. Bei den relativ niedrigen Leitfähigkeiten von 180-430 $\mu \mathrm{S} / \mathrm{cm}$ kann auch ohne weitergehende Isotopenanalyse von überwiegend meteorisch geprägten Wässern ausgegangen werden. Alle Quellproben weisen Sauerstoffgehalte zwischen 6,5 und $10,5 \mathrm{mg} / \mathrm{l}$ auf. Das korrigierte Redoxpotenzial $\left(\mathrm{E}_{\mathrm{H}^{-}}\right.$ Wert) liegt für alle Wässer oberhalb von $400 \mathrm{mV}$ und die pH-Werte zwischen 6,8 und 7,9. Das Redoxmilieu ist daher durchgängig als oxidierend anzusehen. Gemäß Klassifizierung nach Furtak \& Langguth sind die Quellwässer als erdalkalisch-hydrogencarbonatisch zu typisieren.

Von den insgesamt 13 beprobten oberflächlichen Quellaustritten wiesen sechs Vanadiumkonzentrationen unterhalb der Nachweisgrenze von $0,03 \mu \mathrm{g} / \mathrm{l}$ auf, sieben jedoch erhöhte Vanadiumwerte mit einem Maximum von $28 \mu \mathrm{g} / \mathrm{l}$ und ei- 
Abb. 2 Probenlokationen der entnommenen Gesteinsproben Fig. 2 Sample locations of the rock samples

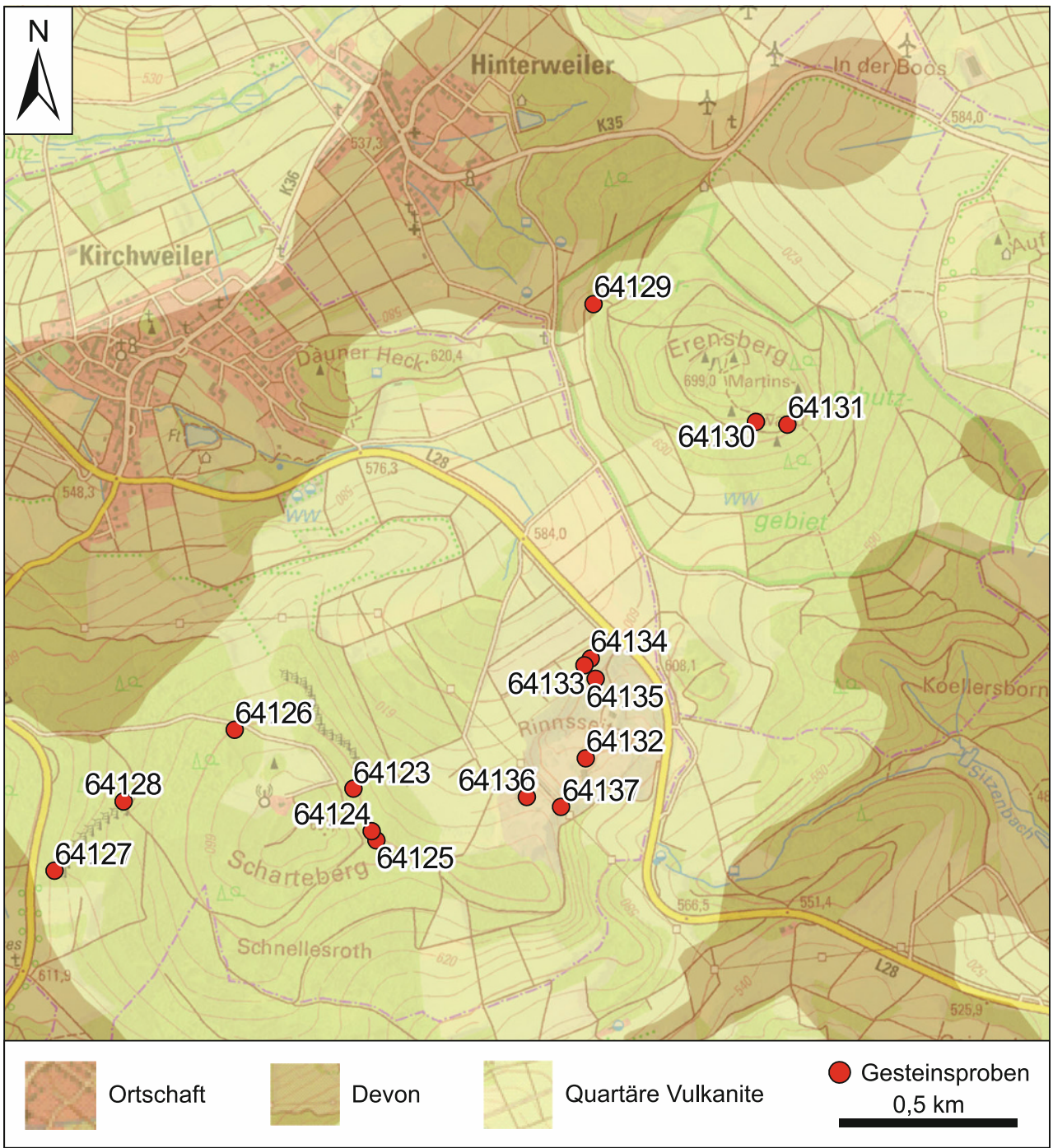

nem Durchschnitt von $17 \pm 10 \mu \mathrm{g} / \mathrm{l}$ (Tab. 1). Alle V-führenden Quellen liegen in Bereichen der Känozoischen Vulkanite (Abb. 1 zeigt dies nur grobmaßstäblich, da hier lateral und vertikal ein schneller Wechsel erfolgen kann), während an den Quellen mit devonischem Umgebungsgestein kein Vanadium $(<0,03 \mu \mathrm{g} / \mathrm{l})$ festgestellt werden konnte. Die niedrigen $\mathrm{V}$-Konzentrationen gehen einher mit niedrigen Phosphatwerten, während die erhöhten V-Konzentrationen linear positiv mit den dann ebenfalls erhöhten Phosphatwerten korrelieren $\left(\mathrm{R}^{2}=0,90\right)$. Die Steigung der Trendlinie entspricht einem stöchiometrischen V/P-Molverhältnis von 0,1 (Abb. 3). Bemerkenswert niedrig waren dagegen die Konzentration von Arsen von meist $<1 \mu \mathrm{g} / \mathrm{l}$ (Härter 2018). Arsen kann zusammen mit Vanadium auftreten und weist dann oft eine Korrelation auf (Wällstedt et al. 2010), nicht jedoch im vorliegenden Fall.

Vanadium liegt im aquatischen Milieu überwiegend als fünfwertige Spezies vor, es sind aber auch vier- und dreiwertige Vanadiumspezies sowie Polymetallatspezies bekannt (Gustafsson 2019). Die Oxidationsstufe bestimmt die Mobilität des Vanadiums, wobei mit zunehmender Oxidationsstufe die Mobilität ansteigt. Bei den oxidierenden Bedingungen der untersuchten Grundwässer werden vor allem $\mathrm{V}(\mathrm{V})$-Spezies erwartet. Zur Validierung dieser Hypothese wurde das pe-pH Prädominanzdiagramm für die V-Spezies mithilfe des USGS-Programms PhreePlot konstruiert (Abb. 4). Die darin geplotteten roten Punkte repräsentieren alle Wasserproben mit erhöhten Vanadiumkonzentrationen. Demnach werden die Wässer durch das Vanadat(V)-Oxoanion $\mathrm{H}_{2} \mathrm{VO}_{4}{ }^{-}$dominiert. Eine Korrelation zwischen Vanadiumkonzentration und $\mathrm{pH}$-Wert ist nicht gegeben und das Auftreten reduzierter Vanadium(III)-Spezies nicht belegbar.

\section{Gesteinsproben}

Die untersuchten mafischen Vulkanitproben aus dem Randbereich des Trockenmaares weisen Vanadiumgehalte von 


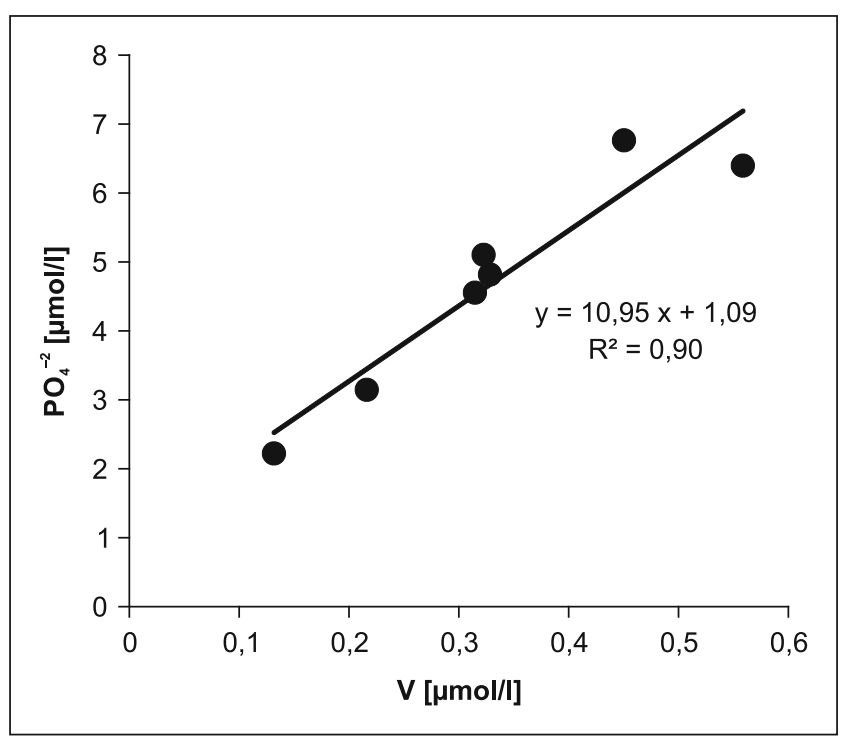

Abb. 3 Die V-Konzentrationen korrelieren linear positiv mit den ebenfalls erhöhten Phosphatwerten. Die Steigung der Trendlinie entspricht einem stöchiometrischen V/P-Molverhältnis von 0,1

Fig. 3 The $\mathrm{V}$ concentrations in the groundwater show a positive linear correlation with phosphate values. The slope of the trend line corresponds to a molar $\mathrm{V} / \mathrm{P}$ ratio of 0.1

200 bis $400 \mathrm{mg} / \mathrm{kg}$ auf. Die im Lavasandtagebau Ringseitert entnommenen Proben zeigen z.B. Werte über $200 \mathrm{mg} / \mathrm{kg}$. Der höchste Gehalt wurde mit $392 \mathrm{mg} / \mathrm{kg}$ ermittelt. Alle mittels RFA analysierten Werte sind der Tab. 2 zu entnehmen. Ein Vergleich mit den mittleren Vanadiumgehalten der oberen Erdkruste von $100 \mathrm{mg} / \mathrm{kg}$ (Gustafsson 2019) weist auf eine geogene Anomalie in der Region mit bis zu 4-fach erhöhten Gehalten in den Vulkaniten auf. Etwa $14 \mathrm{~km} \mathrm{SW}$ der Ringseitert-Struktur in der streichenden Fortsetzung des Rhenohercynischen Gebirgszuges wurde im Rahmen eines Geothermie-Projektes im Jahr 2006 eine Probe entnommen und geochemisch im Labor des LGB Mainz analysiert. Da in dieser Probe lediglich $60 \mathrm{mg} / \mathrm{kg} \mathrm{V}$ gemessen wurden, gibt es keine Anzeichen für eine V-Anreicherung in den devonischen Umgebungsgesteinen.

In Abb. 5 sind die Ergebnisse der Elutionsuntersuchung aufgetragen. Da auch aus der laborreinen Quarzprobe etwas Vanadium eluiert wurde, kann eine geringfügige Kontamination in der Mühle nicht ausgeschlossen werden. Die eluierbaren Vanadiumanteile der Gesteinsproben lagen bei 0,1-0,3\% des Gesamtgehaltes, mit Ausnahme der Probe Nr. 64128 , bei der mit bis zu $350 \mu \mathrm{g} / 1$ etwa $1 \%$ eluierbar war (Abb. 5). Die Proben 64133-I und 64133-II aus dem gleichen Gestein zeigen den Effekt der Korngröße auf die Eluierbarkeit. Die Probe 64133-I hat eine spezifische Oberfläche von $12,5 \mathrm{~m}^{2} / \mathrm{g}$, während die Probe 64133 -II lediglich $7,8 \mathrm{~m}^{2} / \mathrm{g}$ aufweist. Dementsprechend fast doppelt so hoch war die Eluierbarkeit der Probe 64133-I. Generell wurde im zweiten Versuchsdurchlauf (96h) etwa ebenso viel eluiert wie im ersten Versuchsdurchlauf nach $24 \mathrm{~h}$, was auf

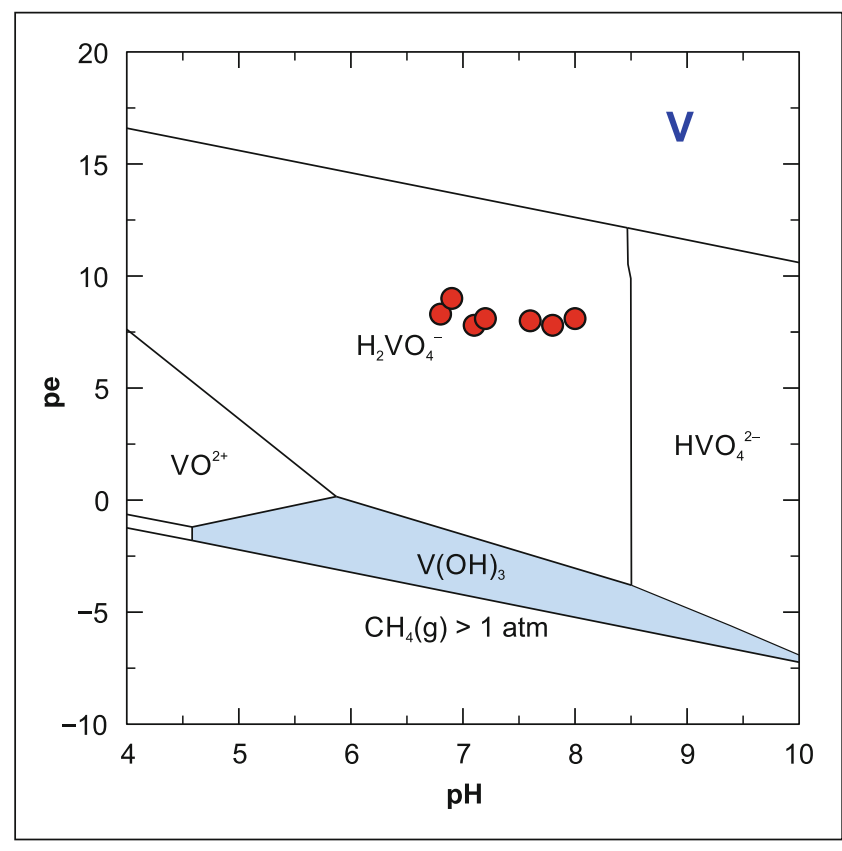

Abb. 4 Das pe/pH-Diagramm der Vanadiumspeziation für die Quellproben. Die gelösten V(IV)- und V(V)-Spezies sind in den weißen Feldern, das schwerlösliche V(III)-Hydroxid als untere Begrenzung im blauen Feld. Die roten Punkte markieren den pe/pH-Verteilungsbereich der Quellwasserproben. Das Diagramm wurde mit Hilfe des USGSProgramms PhreePlot (Kinniburgh und Cooper 2018) sowie den aktuellen thermodynamischen Konstanten von Gustafsson (2019) erstellt

Fig. 4 A pe-pH diagram of the vanadium speciation for the spring water samples. The dissolved V(IV) and V(V) species are in the white fields, the sparingly soluble V(III) hydroxide as the lower bound in the blue field. The red dots mark the $\mathrm{pe} / \mathrm{pH}$ distribution range of the spring water samples. The diagram was prepared using the USGS program PhreePlot (Kinniburgh and Cooper 2018) and using thermodynamic constants recently compiled by Gustafsson (2019)

eine Gleichgewichtsaufsättigung hinweist. Die eluierbaren Gehalte waren in allen Fällen deutlich höher als die Konzentrationen in den Wasserproben und weisen daher auf relativ hohe potenziell mobilisierbare Vanadiumkonzentrationen hin. Letztlich war aber keine direkte Korrelation zwischen den Vanadiumkonzentrationen in den Elutionsproben und den Vanadiumgehalten der aufbereiteten Gesteinsproben festzustellen. Es konnte aber mit dem Versuch zumindest eindeutig nachgewiesen werden, dass das geogene Vanadium eluierbar war.

Zur weiteren Differenzierung möglicher Wirtsphasen für das Vanadium wurde mittels Polarisationsmikroskopie der Mineralbestand der Vulkanite und der verfestigten Lapillilagen analysiert. Alle basaltischen Gesteinsproben weisen ein porphyrisches Gefüge mit Einsprenglingen in der Grundmasse auf. Als dominanter Mineralbestand der Einsprenglinge traten Olivin, Klinopyroxen (Augit), Nephelin/Leucit sowie fluidal eingeregelte, leistenförmige Plagioklase auf. Akzessorisch traten Quarz, Magnetit, Olivin, Biotit, Titanit und Titanaugit sowie Apatit auf. Prinzipiell können die Ge- 
Tab. 2 Vanadiumgehalte der entnommenen Gesteinsproben Table 2 Vanadium contents of the rock samples

\begin{tabular}{llll}
\hline Proben-Nr & Vanadiumgehalt $[\mathrm{mg} / \mathrm{kg}]$ & Proben-Nr & Vanadiumgehalt $[\mathrm{mg} / \mathrm{kg}]$ \\
\hline 64123 & 370 & 64131 & 336 \\
64124 & 334 & 64132 & 284 \\
64125 & 296 & 64133 & 338 \\
64126 & 297 & 64134 & 265 \\
64127 & 392 & 64135 & 344 \\
64128 & 289 & 64136 & 318 \\
64129 & 312 & 64137 & 217 \\
64130 & 343 & - & - \\
\hline
\end{tabular}

halte an dreiwertigem Eisen in einigen der o.g. mafischen Minerale durch dreiwertiges Vanadium substituiert werden, da deren Ionenradien nahezu gleich sind $\left(\mathrm{Fe}^{+3} 65 \mathrm{pm}, \mathrm{V}^{+3}\right.$ 64 pm, Huang et al. 2015). Zur Verifizierung dieser Hypothese wurden Mikrosondenanalysen an Gesteinsdünnschliffen durchgeführt, die jedoch keine Korrelation zwischen Fe- und V-reichen, wohl aber eine auffällige Korrelation zwischen P- und V-reichen Spots ergaben. An drei solcher Mineralkörner wurde schließlich eine chemische Mikroanalyse mithilfe der wellenlängendispersiven Detektoren der Mikrosonde durchgeführt (Abb. 6), die einen Elementgehalt (in Oxidnotation) von $54 \pm 2$ Gew.- $\% \mathrm{CaO}, 36 \pm 1$ Gew.- $\%$ $\mathrm{P}_{2} \mathrm{O}_{5}$ sowie jeweils $5 \pm 0,5$ Gew. $\% \quad \mathrm{~V}_{2} \mathrm{O}_{3}$ und $\mathrm{F}$ ergaben. Diese Zusammensetzung ist kompatibel mit dem Mineral Fluorapatit, bei dem ein Teil (10\%) des Phosphates durch Vanadat substituiert ist. Diese Substitution wird durch die Isotypie beider Oxoanionen $\mathrm{PO}_{4}{ }^{-3}$ und $\mathrm{VO}_{4}{ }^{-3}$ erleichtert.
Fluorapatit kann daher eine ideale Mischkristallreihe mit der Stöchiometrie $\mathrm{Ca}_{5}\left(\mathrm{P}_{\mathrm{x}} \mathrm{V}_{1-\mathrm{x}} \mathrm{O}_{4}\right)_{3}(\mathrm{OH}, \mathrm{F})$ ausbilden (Onda et al. 2008). Dieses $\mathrm{P} / \mathrm{V}$-Molverhältnis entspricht wiederum der stöchiometrischen Sättigungskurve der Wasserproben (Abb. 3). Es erscheint naheliegend, den akzessorisch in allen Gesteinsproben feinkörnig auftretenden Apatit als mögliche, wenn nicht sogar primäre geogene Vanadiumquelle anzunehmen.

Eine Literaturrecherche ergab zwar keine Publikation zu thermodynamischen Löslichkeitskonstanten für V-führenden Fluorapatit. Gupta et al. (1987) geben aber wässrige Löslichkeitswerte von $0,01 \mathrm{mmol} / 1$ für Vanadium, $0,05 \mathrm{mmol} / 1$ für Phosphat sowie $0,1 \mathrm{mmol} / 1$ für Calcium aus der stöchiometrischen Auflösung von Hydroxyapatit der Zusammensetzung $\mathrm{Ca}_{5}\left(\mathrm{P}_{0.83} \mathrm{~V}_{0.17} \mathrm{O}_{4}\right)_{3}(\mathrm{OH})$ bei einem pH 7,1 an. Die Löslichkeitskonstante von Fluorapatit $\left(\log K_{\mathrm{sp}}=55,7\right)$ ist zwar um zwei Größenordnungen ge-

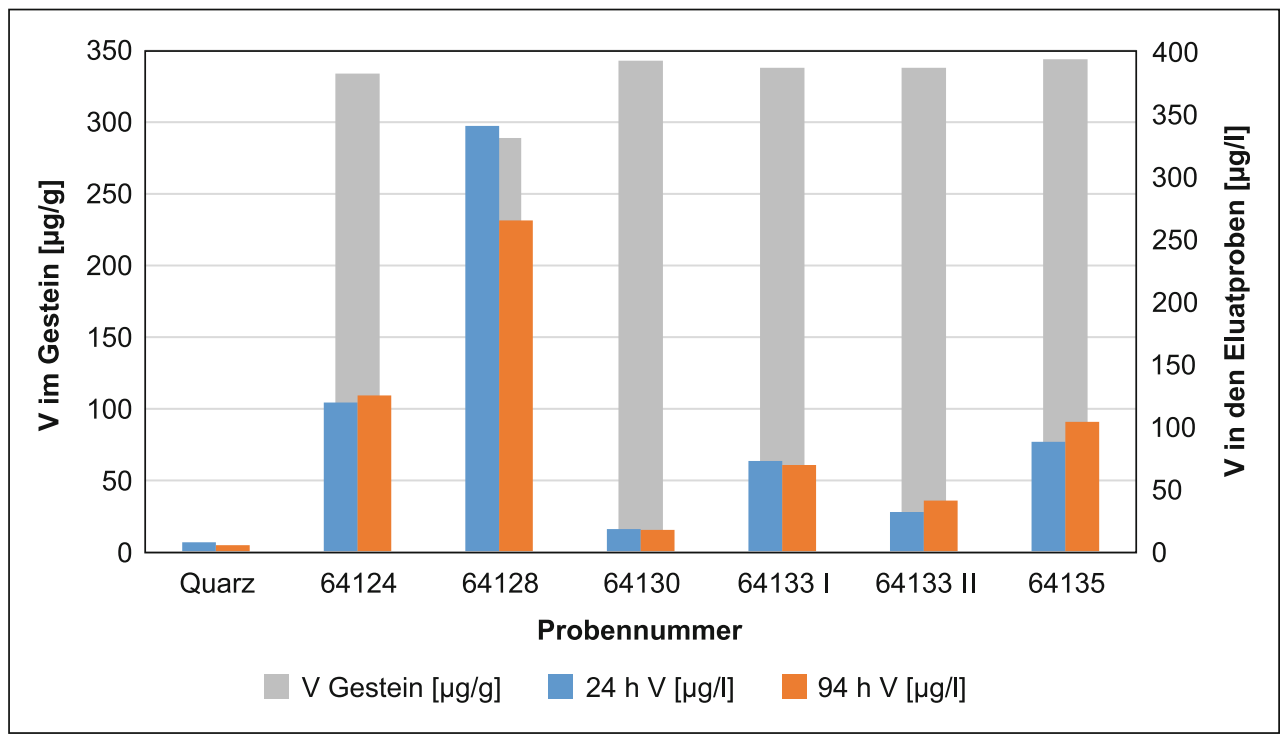

Abb. 5 Ergebnis der Elutionsversuche nach DIN 19529. Die Nummerierung der Gesteinsproben entspricht den Lokationen in Abb. 2. Die blauen Balken zeigen die eluierbaren Vanadiumkonzentrationen nach $24 \mathrm{~h}$, die orangenen Balken die nach weiteren $94 \mathrm{~h}$. Die Vanadiumkonzentration des jeweiligen Eluates ist der rechten Ordinate zu entnehmen. Die grauen Balken zeigen die in dem Gestein enthaltenen Gesamtgehalte in mg/kg (linke Ordinate). Es sei explizit auf den Faktor 1000 zwischen den beiden Konzentrationsskalen hingewiesen ( $\mu \mathrm{g} / \mathrm{l} \mathrm{vs} . \mathrm{mg} / \mathrm{kg}$ )

Fig. 5 Results of the elution experiments with rock powder by means of shaking tests according to DIN 19529. The sample numbers correspond to the locations in Abb. 2. The blue bars show the mobilisable vanadium concentrations after $24 \mathrm{~h}$, the orange bars after another $94 \mathrm{~h}$. The vanadium concentration of the respective eluate can be taken from the right axis. The gray bars show the total contents contained in the rock in $\mathrm{mg} / \mathrm{kg}$ (left axis). Note the factor of 1000 between the two concentration scales $(\mu \mathrm{g} / \mathrm{lvs} . \mathrm{mg} / \mathrm{kg}$ ) 


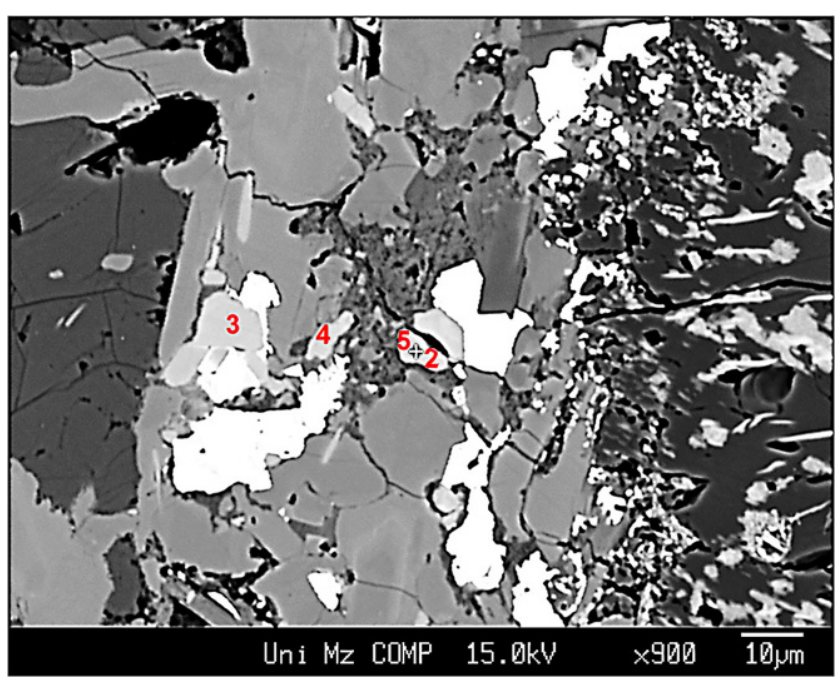

Abb. 6 Mikrosondenbild einer Vulkanitprobe aus dem RingseitertTrockenmaar (Nr. 64127 in Abb. 2). Der Maßstab ist rechts unten zu finden. Generell gilt für dieses BSE-Bild, das je heller das jeweilige Mineralkorn erscheint, desto schwerer sind die darin enthaltenen Elemente. Die nahezu weißen Körner enthalten viel Eisen, die dunklen Bereiche sind Alkali- und Erdalkalihaltige Silikate. Die vier rot markierten Bildpunkte in drei Mineralkörnern mittlerer Graustufe von je etwa $10 \mu \mathrm{m}$ Größe ergaben eine chemische Zusammensetzung von $54 \pm 2$ Gew.- $\% \mathrm{CaO}, 36 \pm 1$ Gew. $\% \mathrm{P}_{2} \mathrm{O}_{5}$ sowie jeweils $5 \pm 0,5$ Gew. $-\%$ $\mathrm{V}_{2} \mathrm{O}_{3}$ und $\mathrm{F}$ (in Oxid-Notation wie für RFA-Analysen üblich)

Fig. 6 Microprobe image of a volcanic sample from the Ringseitert-Trockenmaar (No. 64127 in Abb. 2). The scale can be found on the bottom right. In general for this BSE image, the brighter the respective mineral grain appears to be, the heavier the elements contained within it. The almost white grains contain iron, the dark areas are alkali and alkaline earth silicates. The four red-marked pixels in three mineral grains of average gray scale, each about $10 \mu \mathrm{m}$ in size, gave a chemical composition of $54 \pm 2$ wt. $-\% \mathrm{CaO}, 36 \pm 1$ wt. $-\% \mathrm{P}_{2} \mathrm{O}_{5}$ and $5 \pm 0.5$ wt. $-\%$ $\mathrm{V}_{2} \mathrm{O}_{3}$ and $\mathrm{F}$ (in oxide notation as usual for RFA analysis)

ringer als die von Hydroxyapatit $\left(\log K_{\mathrm{sp}}=53,3\right.$; Zhu et al. 2009). Die Phosphat- und Ca-Konzentrationen zwischen beiden Apatitphasen werden aber vergleichbar, da die von Zhu et al. (2009) berichtete Gleichgewichtskonzentration für Fluorid von $60 \mu \mathrm{mol} / 1$ um zwei Größenordnungen höher liegt als die $\mathrm{OH}^{-}$-Ionenkonzentration bei einem $\mathrm{pH} 7,1$. Da zudem die Ca-Konzentrationen im Grundwasser durch leichter lösliche Phasen wie Calcit bestimmt werden und daher ebenfalls um den Faktor 10 höher sind, würden sich die P- und V-Konzentrationen dementsprechend um den Faktor 10 verringern und dann den gemessenen Werten in etwa entsprechen (Tab. 1).

\section{Schlussfolgerungen}

In Vulkanitproben der Westeifel wurden erhöhte geogene Vanadiumgehalte festgestellt. Elutionsuntersuchungen dieser Proben weisen auf eine gleichgewichtskontrollierte Mobilisierbarkeit des Vanadiuminventars hin. Dementspre- chend wurden in natürlich zutagetretenden Quellwasserproben eines Trockenmaares um eine Größenordnung über dem LAWA-Geringfügigkeitsschwellenwert erhöhte Vanadiumkonzentrationen gefunden. Da die Überschreitungen eine natürliche Ursache haben, gelten die Schwellenwerte trotzdem als eingehalten (GrwV § 7, Abs. 3 letzter Satz), und es sind keine Maßnahmen zur Verminderung der Belastung erforderlich (und möglich). Zur Identifizierung der Quelle für die Vanadiumanomalie wurden Mikrosondenanalysen an einer Vulkanitprobe durchgeführt, die feinkörnigen Vanadat-führenden Fluorapatit als geogene Quelle belegen. Das P/V-Molverhältnis in den Wasserproben entspricht dem Verhältnis aus den Mikrosondenuntersuchungen der Apatitkörner in der Vulkanitprobe und deutet auf eine Gleichgewichtsreaktion hin. Dementsprechend ergab eine hydrogeochemische Gleichgewichtsmodellierung die Oxoanionen $\mathrm{H}_{2} \mathrm{VO}_{4}{ }^{-}$und $\mathrm{HVO}_{4}{ }^{-2}$ des fünfwertigen Vanadiums als dominierende V-Spezies in den Quellwasserproben.

Trockenmaare haben im eher grundwasserarmen Schiefergebirge eine wichtige wasserwirtschaftliche Bedeutung. Aufgrund der Stichtagsmessungen im Rahmen dieser zeitlich begrenzten Studie ist eine umfassende Bewertung der in der Westeifel herrschenden Begebenheiten nicht möglich. Bedingt durch den ähnlichen geogenen Hintergrund ist zu befürchten, dass auch in anderen Grundwasservorkommen als dem des beprobten Trockenmaares der Vulkaneifel erhöhte Vanadiumkonzentrationen auftreten könnten. Da mindestens ein Viertel der in der Westeifel befindlichen Trockenmaare wasserwirtschaftlich genutzt werden kann, sollte Vanadium in die operative Überwachung der Trinkwasserversorgung einbezogen werden, wobei im Falle erhöhter Werte eine Trendumkehr aufgrund des geogenen Ursprungs sowie der relativ geringen Filterwirkung der Vulkanite nicht zu erwarten ist. Falls im Zuge vorsorgender Maßnahmen entschieden wird, das Vanadium bei der Trinkwasseraufbereitung zu entfernen, werden in der Literatur Festbettfilteranlagen auf Eisenoxidbasis als hinreichend effizient empfohlen (Bahr et al. 2019).

Danksagung Wir danken den Verbandsgemeindewerken Daun, insbesondere Herrn Bürgermeister Werner Klöckner sowie dem stellvertretenden Werksleiter Herrn Klaus-Willi Wirtz, für die Unterstützung bei der Probennahmekampagne sowie die Zustimmung zur Publikation dieser Studie.

Funding Open Access funding provided by Projekt DEAL.

Open Access Dieser Artikel wird unter der Creative Commons Namensnennung 4.0 International Lizenz veröffentlicht, welche die Nutzung, Vervielfältigung, Bearbeitung, Verbreitung und Wiedergabe in jeglichem Medium und Format erlaubt, sofern Sie den/die ursprünglichen Autor(en) und die Quelle ordnungsgemäß nennen, einen Link zur Creative Commons Lizenz beifügen und angeben, ob Änderungen vorgenommen wurden.

Die in diesem Artikel enthaltenen Bilder und sonstiges Drittmaterial unterliegen ebenfalls der genannten Creative Commons Lizenz, sofern 
sich aus der Abbildungslegende nichts anderes ergibt. Sofern das betreffende Material nicht unter der genannten Creative Commons Lizenz steht und die betreffende Handlung nicht nach gesetzlichen Vorschriften erlaubt ist, ist für die oben aufgeführten Weiterverwendungen des Materials die Einwilligung des jeweiligen Rechteinhabers einzuholen.

Weitere Details zur Lizenz entnehmen Sie bitte der Lizenzinformation auf http://creativecommons.org/licenses/by/4.0/deed.de.

\section{Literatur}

Arena, G., Copat, C., Dimartine, A., Grasso, A., Fallico, R., Sciacca, S., Fiore, M., Ferrante, M.: Determination of total vanadium and vanadium $(\mathrm{V})$ in groundwater from Mt. Etna and estimate of daily intake of vanadium (V) through drinking water. J. Water Health 13, 522-530 (2015)

Bahr, C., Payer, H., Simon, A.: Entfernung von Vanadium aus Trinkwasser mit granuliertem Eisenhydroxid: Ergebnisse eines Pilotversuchs im Saarland. GWF-Wasser 06/2019., S. 79-88 (2019)

Bergmann, S., Fritsche, J.G., Quadflieg, A., Richts, A., Wagner, B., Walter, T., Wolter, R.: Hydrogeochemische Hintergrundwerte im Grundwasser und ihre Bedeutung für die Wasserwirtschaft. Länderarbeitsgemeinschaft Wasser (LAWA), Bund-Länder-Ausschuss Bodenforschung (BLA-GEO), BGR, Hannover (2015). 22 $\mathrm{S}$

Burri, N.M., Weatherl, R., Moeck, C., Schirmer, M.: A review of threats to groundwater quality in the anthropocene. Sci. Total Environ. 684, 136-154 (2019)

Donndorf, S.: Geowissenschaftliche Untersuchung der RingseitertVulkangruppe (Kirchweiler/Westeifel). Diplomarbeit. FriedrichSchiller-Universität Jena, Jena (2009). $71 \mathrm{~S}$

Gupta, S.K., Rao, P.V.R., George, G., Narasaraju, T.S.B.: Determination of solubility products of phosphate and vanadate apatites of calcium and their solid solutions. J. Mater. Sci. 22, 1286-1290 (1987)

Gustafsson, J.P.: Vanadium geochemistry in the biogeosphere-speciation, solid-solution interactions, and ecotoxicity. Appl. Geochem. 102, $1-25$ (2019)

Hamada, T.: High vanadium content in Mt. Fuji groundwater and its relevance to the ancient biosphere. In: Nriagu, J.O. (Hrsg.) Vanadium in the environment. Part 1: chemistry and biochemistry, S. 97-123. Wiley \& Sons, New York (1998)

Härter, M.L.: Zum Vorkommen von Vanadium in Wässern bei Kirchweiler (Vulkaneifel). Masterarbeit. Johannes Gutenberg-Universität, Mainz (2018). 143 S

Hesse, G.: Hydrogeologische Erkundung von Maar-Diatrem-Vulkanen am Beispiel des Geeser Maares, Westeifel. Dissertation. Friedrich-Schiller-Universität, Jena (2002). $135 \mathrm{~S}$

Huang, J.H., Huang, F., Evans, L., Glasauer, S.: Vanadium: global (bio)geochemistry. Chem. Geol. 417, 68-89 (2015)

Hyon, H.H., Yoon, C.S., Yoon, S.T., Kim, H.R.: Vanadium leaching characteristics of soils originating from basalt basins in Jeju Island. J. Korean Environ. Sci. Soc. 25, 1541-1554 (2016)
Kinniburgh, D.G., Cooper, D.M.: PhreePlot—creating graphical output with phreeqc. 598 S. http://www.phreeplot.org (2018)

Koh, D.-C., Chae, G.-T., Ryu, J.-S., Lee, S.-G., Ko, K.-S.: Occurrence and mobility of major and trace elements in groundwater from pristine volcanic aquifers in Jeju Island, Korea. Appl. Geochem. 65, 87-102 (2016)

Köppen, K.-H.: Geologie und Hydrogeologie der Gerolsteiner Mulde (linksrheinisches Schiefergebirge) unter Berücksichtigung der Mineralwasservorkommen. Mainz. Geowiss. Mitt. 20, 43-54 (1991)

Länderarbeitsgemeinschaft Wasser: LAWA: Ableitung von Geringfügigkeitsschwellenwerten für das Grundwasser. Länderarbeitsgemeinschaft Wasser, Düsseldorf (2004). 33 S

Länderarbeitsgemeinschaft Wasser: LAWA: Ableitung von Geringfügigkeitsschwellenwerten für das Grundwasser Bd. 2016. Länderarbeitsgemeinschaft Wasser, Stuttgart (2017). $28 \mathrm{~S}$

Landesamt für Umwelt, Wasserwirtschaft und Gewerbeaufsicht: LUWG: Hydrogeologischer Atlas Rheinland-Pfalz (Kartenblätter Ausgabe 44). Landesamt für Umwelt, Wasserwirtschaft und Gewerbeaufsicht, Mainz (2005)

Lange, T., Lorenz, V., Köppen, K.-H., Büchel, G.: Neue Aspekte zum Vulkanismus der Westeifel. Jber. Mitt. Oberrhein. Geol. Ver. N.F. 101, 227-250 (2019)

Langguth, H.R., Voigt, R.: Hydrogeologische Methoden, 2. Aufl. Springer, Berlin Heidelberg (2004). 1005 S

Minelli, L., Veschetti, E., Giammanco, S., Mancini, G., Ottaviani, M.: Vanadium in Italian waters: monitoring and speciation of V(IV) and V(V). Microchem. J. 67, 83-90 (2000)

Onda, A., Ogo, S., Kajiyoshi, K., Yanagisawa, K.: Hydrothermal synthesis of vanadate/phosphate hydroxyapatite solid solutions. Mater. Lett. 62, 1406-1409 (2008)

Seib, N., Kley, J., Büchel, G.: Identification of maars and similar volcanic landforms in the West Eifel Volcanic Field through image processing of DTM data: efficiency of different methods depending on preservation state. Int. J. Earth Sci. 102, 875-901 (2013)

Wagner, B., Walter, T., Himmelsbach, T., Clos, P., Beer, A., Budziak, D., Dreher, T., Fritsche, H.-G., Hübschmann, M., Marczinek, S., Peters, A., Poeser, H., Schuster, H., Steinel, A., Wagner, F., Wirsing, G.: Hydrogeochemische Hintergrundwerte der Grundwässer Deutschlands als web map service. Grundwasser 16(3), 155-162 (2011)

Wällstedt, T., Björkvald, L., Gustafsson, J.P.: Increasing concentrations of arsenic and vanadium in (southern) Swedish streams. Appl. Geochem. 25, 1162-1175 (2010)

Weiler, H.: Die Bedeutung der quartären Maare in der Westeifel für die Wasserversorgung. Mainz. Geowiss. Mitt. 31, 153-190 (2002)

Zhu, Y., Zhang, X., Chen, Y., Xie, Q., Lan, J., Qian, J., He, N.: A comparative study on the dissolution and solubility of hydroxylapatite and fluorapatite at $25^{\circ} \mathrm{C}$ and $45{ }^{\circ} \mathrm{C}$. Chem. Geol. 268, 89-96 (2009)

Hinweis des Verlags Der Verlag bleibt in Hinblick auf geografische Zuordnungen und Gebietsbezeichnungen in veröffentlichten Karten und Institutsadressen neutral. 